\title{
Analysis of the influence of citizens' altruism on the effectiveness of the socially-optimal actions stimulation system
}

\author{
M I Geraskin ${ }^{1}$ \\ ${ }^{1}$ Samara National Research University, Moscowskoe shosse 34, Samara, Russia, 443086
}

\begin{abstract}
The problem of the development of the state information stimulation system of Russian citizens' socio-optimal actions is considered according to the optimum of collective utility function as criterion. The algorithm of the information system is formed taking into account the conditions of individual rationality, Pareto efficiency and non-manipulability. The algorithm for analyzing the effect of various functions of the probability distribution density of the altruism degree of Russian citizens on the effectiveness of the system for stimulating socially-optimal actions is developed. The simulation of social groups behavior, covering more than ten percent of Russia's economically active population, is confirmed the stability of the stimulation system for citizens' opportunistic behavior.
\end{abstract}

\section{Introduction}

In a transitional economy [1,2] trends of individual rationality are growing in the society. The state develops the moral improving programs $[3,4]$ to overcome this trends. In this case, the purpose of the state is the social effect of citizens' acts, performing on the basis of maximization of collective but not individual utility function, hereinafter referred to as socio-optimal actions. Achieving this purpose requires the involvement in socially useful activity of large group of the population and personified registration of socio-optimal actions. It needs to organize the state information system, based on the information resources of currently working in Russia programs [5-7].

The concept of socio-optimal actions' stimulation provides for the establishment of information system of personified registration of the actions of citizens (hereinafter, agents). The system also includes the distribution of state stimulation fund in the form of incentives between agents according to certain mechanisms. The dynamics of the system is a two-period. In the first period (registration period) performed socio-optimal actions are recorded, and in the end of this period the stimulation fund is distributed. In the next period (period of stimulation) the earlier distributed incentives are used.

In addition to the utilitarian stimulating function information system also solves the problem of the formation of the agent's status in the hierarchy of the citizens, used for non-material motivation. On a longer time horizon, the state's social priorities could changed by varying the attributes of sociooptimal actions and their monetary valuation can be varied as a result of inflation. Therefore, to comparability of agents' statuses the system accumulates not only incentives as the current cash equivalent of social activity, but also agents' rating in comparable dimension.

The object of stimulation is socio-optimal actions of citizens, that is, actions that correspond to certain attributes. The actions should maximize collective utility function without increasing the individual utility function. Therefore, the attributes correspond to the terms of gratuitousness, public utility and unconnectedness with professional activities of citizens. Consequently, socio-optimal 
actions do not require special qualification, whereby the stimulation object's dimension is duration of action excluding the content of the action. The subject of stimulation is citizen, performing a sociooptimal action in certain period. The apparatus of stimulation is the state represented by certain ministries (departments).

\section{Methods}

The investigations of stimulation systems and distribution mechanisms produce the following mechanisms corresponding the individual rationality. Competitive mechanism is developed with noncooperative [8] and cooperative [9] behavior of agents, its Pareto efficiency and optimality according to additive utility function criterion are proved. The step-by-step resource distribution mechanism (SRDM) is obtained [10], for which proved [11] that nonmanipulability and Pareto efficiency simultaneously only for SRDM; also SRDM, as shown in [12], is equivalent to mechanisms of direct and reverse priorities. It was shown [13] that unique SRDM exists, in which the incentive is distributed [14] as minimum of agent's information and the average undistributed rest of incentives. The approach to the distribution based on the penalty and incentive functions [15] showed the Pareto efficiency and optimality according to additive utility function criterion for compensatory mechanisms; according to a compensatory mechanism incentives are equal to agents' costs. Thus, only SRDM satisfies [16] all above conditions. Since SRDM implies consistent registration of agents' actions and further distribution of the incentives, it is impossible to use in the system, where actions perform independently and record simultaneously with asymmetrical agents' behavior $[17,18]$. The interaction distribution algorithm in a strongly coupled system with a transferable utility is developed $[19,20]$.

On the base of this ideas an algorithm of the information system (Fig. 1) was developed [21]. The algorithm satisfied the conditions of individual rationality, Pareto efficiency, non-manipulability and optimality by the collective utility criterion.

In [19] and later in this paper the following notation is used:

$Z=\left\{z_{i}, i=1, \ldots, I\right\} \quad$ is a set of attributes of socially-optimal actions;

$K(t)=\{1, \ldots, n(t)\}$ is a set of agents, including citizens who perform actions that correspond to the attributes of $Z$; index $n(t)$ denotes the number of agents in a certain period $t$;

$A(Z, t)=\left\{a_{k}(Z, t), k \in K\right\}$ is a vector of socially-optimal actions; the vector includes quantitative estimations of the actions of the $k$-th agent corresponding to the attributes $Z$ in the $t$-th period; estimation $a$ is expressed in the time spent by the agent for performing these actions; the vector belongs to the allowable set $\bar{A}=\left\{a_{k} \in\left[0, a^{\max }\right], a^{\max }>0, k \in K\right\}$, where the symbol $a^{\max }$ denotes the upper limit of the agents' available time;

$F(t) \in\left(0, F^{\max }\right], F^{\max }>0$ is a stimulation fund in the $t$-th period $t \in(0, T]$; further, the index $t$ is omitted, all parameters of the model correspond to a certain period of time;

$$
u_{k}=\psi\left(a_{k}\right), k \in K, \quad \psi\left(a_{k}\right)=\alpha a_{k}^{\beta}, \alpha \in\left(0, \alpha^{\max }\right], \beta \in\left(0, \beta^{\max }\right], \beta^{\max } \in(0,1], k \in K \quad \text { is dimensionless }
$$

function of registration of socially-optimal actions; where $u$ is a score, $\alpha, \beta$ are constant coefficients;

$$
\begin{aligned}
& E=\sum_{k \in K} a_{k} \text { is a system effectiveness indicator; } \\
& x_{k}=\varphi\left(u_{k}\right)=\varphi^{\min }+\left(b_{1}-b_{2} \sum_{k \in K} u_{k}\right) a_{k}, k \in K \quad \text { is monetary incentive function; where } \varphi^{\min } \text { is }
\end{aligned}
$$

guaranteed incentive of non-zero agent's action, $b_{1}, b_{2}>0$ are coefficients of the incentive function, calculated from formulas

$$
b_{1}=\frac{F-n \varphi^{\min }}{\sum_{k \in K} u_{k}} \frac{2 \bar{u}+\sum_{k \in K} u_{k}}{2 \bar{u}}, b_{2}=\frac{F-n \varphi^{\min }}{2 \bar{u} \sum_{k \in K} u_{k}}, \bar{u}=\frac{1}{n} \sum_{k \in K} u_{k} .
$$

The algorithm of a one-period cycle of the information system is shown in Fig. 1. 


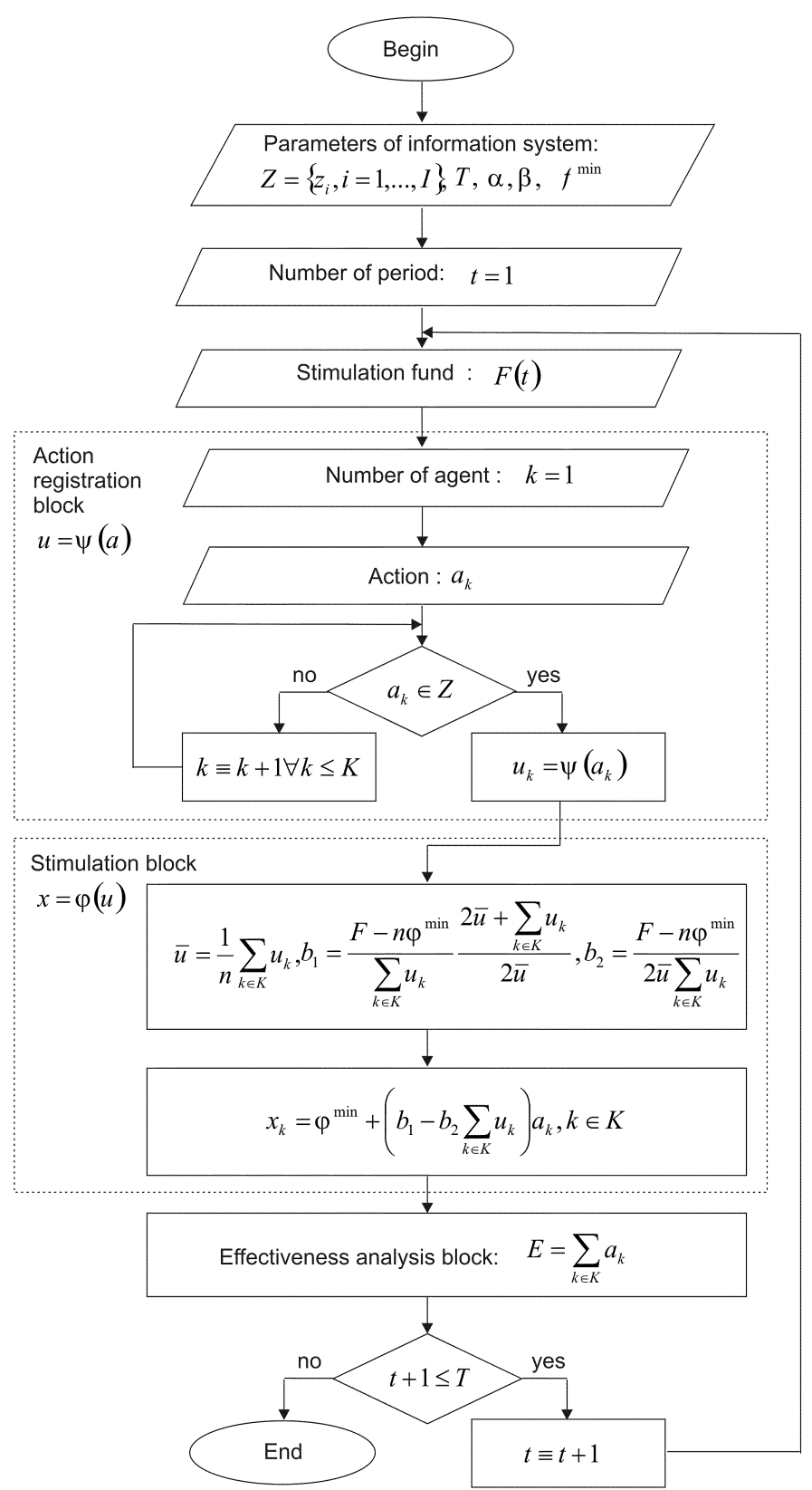

Figure 1. Algorithm of information system cycle.

The action registration block identifies agents' actions in the scoring. The stimulation block is intended for distribution of the stimulation fund depending on the vector of scoring actions. The effectiveness analysis block controls the dynamics of the change in the social efficiency criterion of the system in the selected time interval until the maximum number of work periods is reached.

Let us consider the problem of the information system algorithm modeling for various probability density functions of the existence of social groups with a greater or lesser inclination for altruism.

\section{Results and discussion}

Let the following continuous functions are defined for the $k$-th agent

$$
a_{k}\left(D_{k}\right)=D_{k}^{\delta_{a k}}, d_{k}\left(D_{k}\right)=D_{k}^{\delta_{d k}}, \delta_{a k}, \delta_{d k} \in[0,1], \delta_{a k}+\delta_{d k}=1, D_{k}>>1, k \in K,
$$


where $d_{k}$ is the working time interval; $D_{k}$ is an available time fund equal to the physical time fund except for the rest time; $\delta_{a k}, \delta_{d k}$ are elasticity coefficients of «charitable» time and working time on $D_{k}$.

The functions (1) express the connection between the time interval of charitable actions, the time interval of working time and the available time fund. The derivatives of the functions (1) decrease with increase of $D_{k}$, expressing the propensity of individuals [22] to increase the rest time with growth $D_{k}$.

Definition: altruism (propensity to charity) of the $k$-th agent is a type of function (1), under which $\delta_{a k}>\delta_{d k}$.

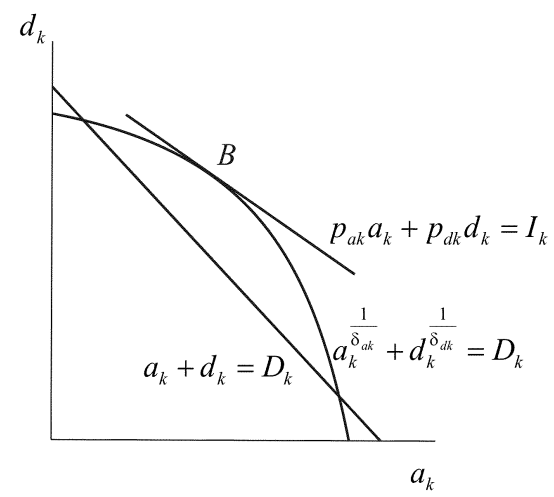

Figure 2. Graphical interpretation of the problem (2), (3).

The introducing of the socially-optimal actions stimulation system by the algorithm (Fig. 1) leads to the fact that altruistic actions bring a non-zero income.

Therefore, to select the value of the action, the $k$-th agent, taking into account the individual rationality, solves the following problem: to maximize the total income from the working and «charitable» time with a restriction ${ }^{1}$ on the available time fund:

$$
\begin{aligned}
\max _{a_{k} \in \bar{A}} I_{k}= & \max _{a_{k} \in \bar{A}}\left(p_{a k} a_{k}+p_{d k} d_{k}\right), p_{a k}, p_{d k}>0, p_{a k}=\frac{x_{k}}{a_{k}}, \\
& a_{k}^{\frac{1}{\delta_{a k}}}+d_{k}^{\frac{1}{\delta_{d k}}}=D_{k}, k \in K,
\end{aligned}
$$

where $p_{a k}$ is the price of the «charitable» time; $p_{d k}$ is the price (tariff rate) of working time.

Note that the restriction (3) does not coincide with the identity $a_{k}+d_{k}=D_{k}$, because of the «charitable» time is not an absolute substitute in relation to working time, as shown in Fig. 2.

The solution of the problem (2), (3) has the form²:

$$
\begin{gathered}
\frac{\delta_{d k}}{\delta_{a k}}\left(D_{k}-a_{k}^{* \frac{1}{\delta_{a k}}}\right)^{\delta_{d k}-1} a_{k}^{* \frac{1}{\delta_{a k}}-1}=\frac{p_{a k}}{p_{d k}}, \\
d_{k}^{*}=\left(D_{k}-a_{k}^{* \frac{1}{\delta_{a k}}}\right)^{\delta_{d k}}, k \in K,
\end{gathered}
$$

${ }^{1}$ The restriction (3) follows from (1): $D_{a k}=a_{k}^{\frac{1}{\delta_{a k}}}, D_{d k}=d_{k}^{\frac{1}{\delta_{d k}}}, D_{a k}+D_{d k}=D_{k}$.

${ }^{2}$ The equation $d_{k}=\left(D_{k}-a_{k}^{\frac{1}{\delta_{a k}}}\right)^{\delta_{d k}}$ follows from (2) ; therefore $d_{k a_{k}}^{\prime}=-\frac{\delta_{d k}}{\delta_{a k}}\left(D_{k}-a_{k}^{\frac{1}{\delta_{a k}}}\right)^{\delta_{d k}-1} a_{k}^{\frac{1}{\delta_{a k}}-1}$; as at the tangency point $B$ the angular coefficients of the functions (2) and (3) are equal, the optimum condition has the form (4). 
where the symbol «*» denotes the optimal values.

The elasticity coefficients $\delta_{a k}, \delta_{d k}$ are known, and price vectors are given

$$
\boldsymbol{P}_{a}=\left\{p_{a k}, k \in K\right\}, \boldsymbol{P}_{d}=\left\{p_{d k}, k \in K\right\},
$$

the first vector is calculated on the basis of the results of algorithm (Fig. 1) by formula (2), and the second vector is given based on the statistics of the national labor market. Thus, the solution of the equation (4) gives an individually rational vector of socially-optimal actions $\boldsymbol{A}$.

Let the coefficient $\delta_{a k}$ in the general population of agents (population) is a random variable [23] with a normal distribution law [21]:

$$
f\left(\delta_{a}\right)=\frac{1}{\sigma \sqrt{2 \pi}} e^{-\frac{w\left(\delta_{a}-\bar{\delta}^{\prime}\right)^{2}}{2 \sigma^{2}}} .
$$

where $\bar{\delta}, \sigma$ are the mathematical expectation and the standard deviation of the initial distribution of the random variable; $l$ is a coefficient taking into account the asymmetry ( $l>1$ - left asymmetry, $l<1$ right asymmetry at $0<\bar{\delta}<1)$ in comparison with the normal law $(l=1)$; $w$ is a coefficient that takes into account kurtosis ( $w<1$ is a more uniform distribution, $w>1$ is a less uniform distribution) compared with the normal law $(w=1)$.

In this case, the expected values of the agents' number having these values of the elasticity coefficients $\delta_{a k}, \delta_{d k}=1-\delta_{a k}$, are calculated by the formulas

$$
\hat{n}\left(\delta_{a}\right)=f\left(\delta_{a}\right) n, \hat{n}\left(\delta_{d}\right)=n-\hat{n}\left(\delta_{a}\right),
$$

where $\hat{n}\left(\delta_{a}\right), \hat{n}\left(\delta_{d}\right)$ are the expected values of the agents' number at given values of the elasticity coefficients.

The algorithm of the effectiveness analyzing block of the information system for various parameters of the probability density function of the social groups presence with a greater or lesser inclination for altruism is shown in Fig. 3.

The simulation of the stimulation effect on the population's behavior is carried out by changing the asymmetry and kurtosis of the probability density function (7) of a normal distribution. The characteristics of the population's distribution variants by social groups with greater or lesser inclination for altruism are given in Table. 1 . The variant $\theta=1$ corresponds to the normal distribution law; variant $\theta=2$ simulates the case of exceeding the mathematical expectation of the population's inclination to altruism over the median value due to right asymmetry; variant $\theta=3$ simulates the case of a more uniform distribution of the population's propensity for altruism in comparison with the normal distribution law due to the decrease in kurtosis.

Table 1. Variants of population's distribution.

\begin{tabular}{lccc}
\hline Model & \multicolumn{3}{c}{ Variant } \\
\cline { 2 - 4 } parameter & $\theta=1$ & $\theta=2$ & $\theta=3$ \\
\hline Characteristics & normal & normal distribution with right & normal distribution with reduced \\
& distribution & asymmetry & excess \\
$l$ & 1 & 0,8 & 1 \\
$w$ & 1 & 1 & 0,5 \\
\hline
\end{tabular}

The following initial data are considered: the value of the stimulation fund is set $F=1000000$ thousand rubles, the values of the registration function coefficients $(\alpha=67, \beta=0,8)$ are selected from the condition $\bar{u}=100$ at the initial value of the time price and $\theta=1$, the minimum stimulus is assumed equal to zero, the number of the population $n=9727$ thousand corresponds to the group of Russian Federation population with income below the subsistence minimum, the price of working time is $P_{d}=$ 0.21 thousand rubles per hour based on the average wage of 35.369 thousand rubles per month, the range of the elasticity coefficient of the «charitable» time is chosen taking into account the possibility 
of equation (4) solution equal to $\delta_{a} \in[0,1 ; 0,7]$. Since $\varphi^{\min }=0$, the price of «charitable» time is the same for all agents and it is equal to $P_{a}=\frac{x_{k}}{a_{k}}=b_{1}-b_{2} \sum_{k \in K} u_{k}$.

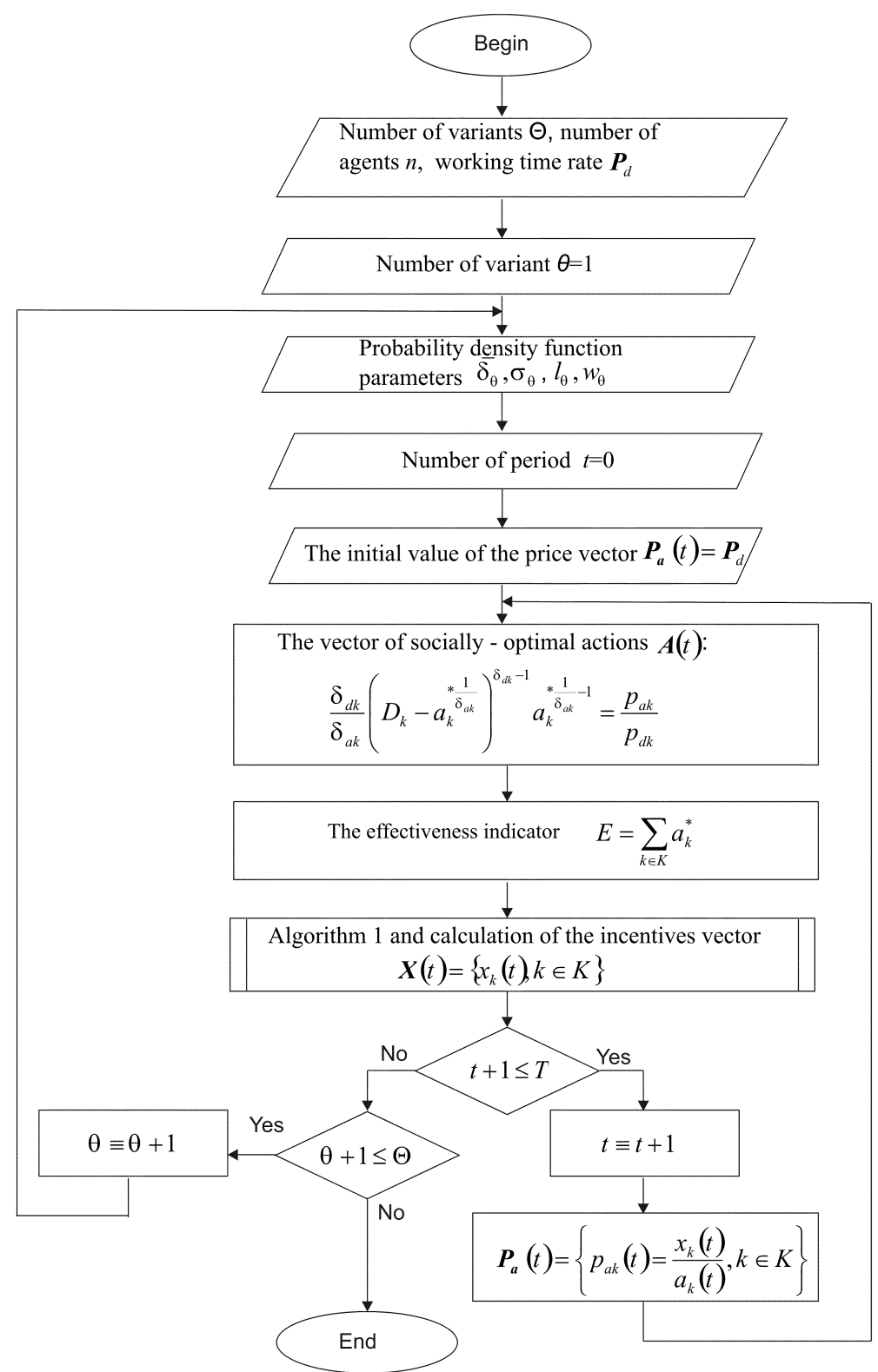

Figure 3. Algorithm of the effectiveness analyzing block of the information system.

In Fig. 4 the distribution functions of the population depict depending on the elasticity coefficient of the «charitable» time for various simulation variants.

In Fig. 5 the functions of average per capita effectiveness indicator $E_{a v .}=E / n$ for different simulation variants are given. The sharp increase in the effectiveness indicator at $t=1$ is due to the increase in the price of the «charitable» time (Fig. 8) in the first period in comparison with the initial date adopted by $P_{a}=P_{d}$ to adjust the stimulation system in the zero period. From Fig. 6 it follows that the reason for this growth is a significant increase in socially-optimal actions in groups with relatively low of «charitable» time elasticity coefficient in the range $(0.2,0.5)$. 

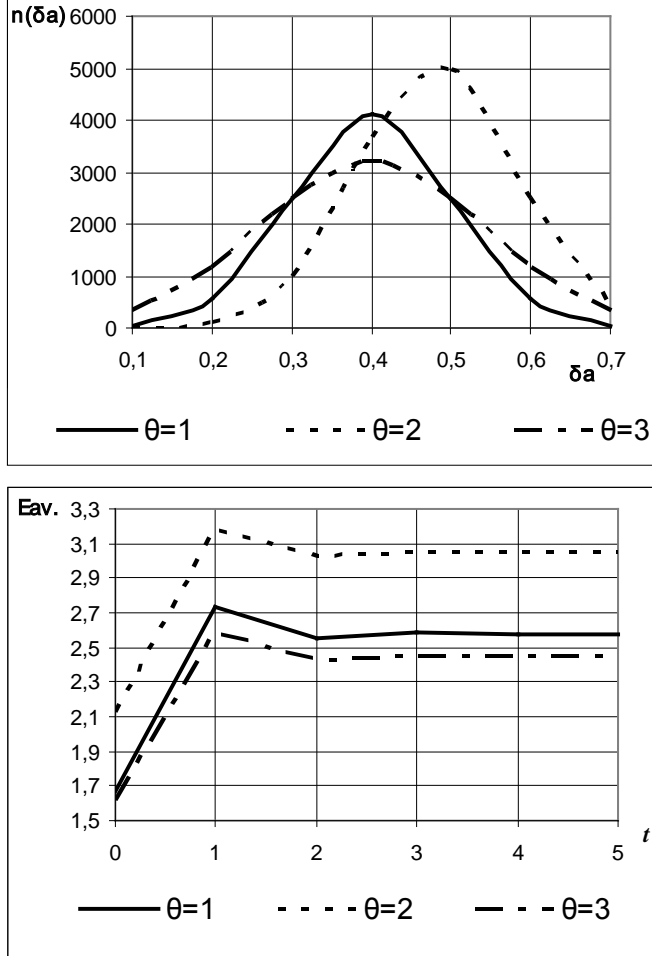

Figure 4. Distribution functions of the population (thousand) depending on the value of the elasticity coefficient of the «charitable» time for various simulation variants.
Figure 5. Dynamics of the average per capita effectiveness indicator for various simulation variants.

Population groups with very low values $(0,1 \ldots 0,2)$ and very high $(0,5,0,7)$ values of the «charitable» time elasticity coefficient are less susceptible to the stimulating effect of the high price $P_{a}$ : the first because of the prevalence of work in their disposable time fund, the latter due to high altruism. Since at $\mathrm{t}=2$, as the price $P_{a}$ decreases (Fig. 8), the effectiveness indicator also decreases taking into account (Fig. 6) sharp reduction of such actions in the range $(0.2,0.5)$. Consequently it can be concluded that population groups with relatively low «charitable» time elasticity coefficient are prone to opportunistic behavior, that is, the motivation for socially-optimal actions for them is individual rationality. As a result, in all variants $\theta=1,2,3$ the value of the average effectiveness indicator stabilizes even at $\mathrm{t}=3$.

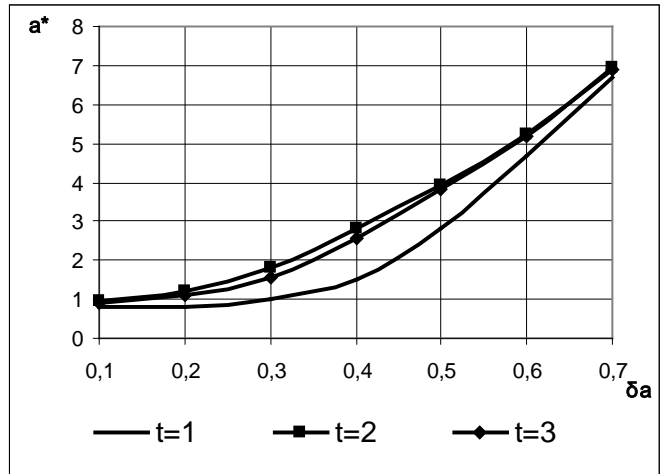

Figure 6. Dependence of sociallyoptimal actions on the elasticity coefficient of the «charitable» time for $\theta=1$.

Stimulation is most effective at $\theta=2$, the least effective at $\theta=3$. Consequently, the results of the simulation are consistent with the following provisions: 1 ) an increase in the prevalence in a society of citizens with a higher propensity for altruism leads to an increase in the social effect from charity stimulating; 2) in case of stimulation a more equal prevalence in a society of individuals with high and low inclination to altruism leads to a reduction in the number of socially optimal actions in comparison with the normal distribution.

In Fig. 7 the average registration score per capita $u_{a v}=\bar{u}$ dependences on the period for different simulation variants are given. In all variants, the value $u_{a v}$ stabilizes at $\mathrm{t}=3$. The highest values of 
average score are recorded at $\theta=2$, the lowest values are recorded at $\theta=3$, which is due to the dependence of the average score through the registration function on the social effect $E_{a v}$.

In Fig. 8 the «charitable» time price dependences on the period for different simulation variants are given, which also stabilize at $\mathrm{t}=3$. The lowest price $P a$ corresponds to $\theta=2$, the highest price is reached in the case $\theta=2$. Since the price $P a$ is a state incentive for a single socially-optimal action, the stimulation system is most economically effective at $\theta=2$, the least effective at $\theta=3$.

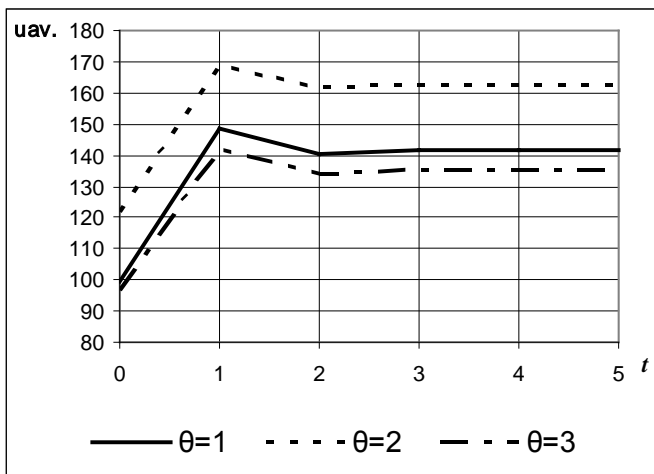

Figure 7. Dynamics of the average score per capita of the registration function for various simulation variants.

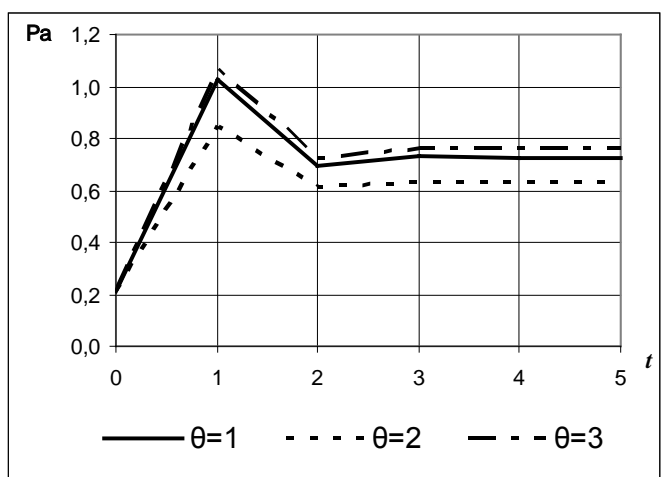

Figure 8. Dynamics of the «charitable» time price (thousand rubles / hour) for various simulation variants.

The stabilization of the effectiveness indicator of the simulation system (Fig. 5) and the price of time (Fig. 8) at $t>2$ leads to the conclusion about the stability of the simulation system for opportunistic behavior of citizens: persons with relatively low values of «charitable» time elasticity, performing socially-optimal actions based on individual rationality, demonstrate the greatest negative sensitivity to the change in the price of time as a stimulus. Due to this, the positive deviation of the stimulus in one period from a certain equilibrium value is damped by the opportunistic behavior of citizens, leading in the next period to a decrease in the number of socially-optimal actions.

\section{Conclusion}

The problem of informational support of the state strengthening morality strategy by stimulating citizens' actions performed on the basis of maximizing the collective utility function is considered. The following main results are obtained in the article.

The algorithm for analyzing the effectiveness of the information simulation system for various parameters of the probability distribution function of social groups with greater or lesser inclination to altruism in society is developed.

The influence of stimulation on the population's behavior is studied by changing the asymmetry and kurtosis of the probability density function of the normal distribution. The case of exceeding the mathematical expectation of the population's inclination to altruism over the median value is simulated by introducing asymmetry. The case of a more uniform distribution of the population's inclination to altruism in comparison with the normal distribution law is simulated by the introduction of kurtosis.

The analysis shows that the stimulation system is resistant to opportunistic citizens' behavior due to the fact that persons performing socially-optimal actions based on the individual rationality demonstrate the greatest negative sensitivity to the change in the stimulus.

\section{References}

[1] Roland G 2000 Transition and Economics. Politics, Markets, and Firms (Cambridge: MIT Press) p 840

[2] Braguinsky S, Yavlinsky G 2000 Incentives and Institutions. Transition to a Market Economy in Russia (NJ.: Princeton University Press) p 420

[3] RF Government Decree of 30.12.2015 N 1493 "On State program" Patriotic Education of Citizens of the Russian Federation for 2016 - 2020" 
[4] RF Government Decree of December 27, 2012 N 2567-r "On the state program of the Russian Federation" Development of Culture and Tourism "2013 - 2020"

[5] RF Government Decree of 15.04.2014 N 313"On approval of the Russian Federation, the state program" Information Society (2011 - 2020)"

[6] RF Government Decree of 27.12.2012 N 1406 "On the federal target program" Development of the Russian judicial system for 2013 - 2020"

[7] RF Government Decree of 15.04.2014 N 320 "On approval of the state program of the Russian Federation" Public Financial Management and regulation of financial markets"

[8] Burkov V N, Danev B, Enaleev A K, Nanev T B, Podvalny L D and Yusupov B S 1988 Competitive mechanisms in problems of distribution of scarce resources Automation and Remote Control 11 142-153

[9] Burkov V N, Enaleev A K and Kalenchuk V F 1989 Coalition with the competitive mechanism of resource distribution Automation and Remote Control 12 81-90

[10] Burkov V N, Enaleev A K and Lavrov Y G 1992 Synthesis of optimal planning and incentive mechanisms in the active system Automation and Remote Control 10 113-120

[11] Burkov V N, Iskakov M B and Korgin N A 2010 Application of generalized median schemes for the construction of non-manipulable mechanism multicriterion active expertise Automation and Remote Control 71(8) 1681-1694

[12] Korgin N A 2009 Equivalence of non-manipulable and non-anonymous priority resource distribution mechanisms Managing large systems $26.1319-347$

[13] Burkov V N, Gorgidze I I, Novikov D A and Yusupov B S 1997 Models and cost and revenue distribution mechanisms in the market economy (Moscow: Institut problem upravleniya) p 356

[14] Korgin N A 2010 Use of intersection property for analysis of feasibility of multicriteria expertise results Automation and Remote Control 71(6) 1169-1183

[15] Chumak V G, Ramzaev V M and Khaimovich I N 2015 Challenges of Data Access in Economic Research based on Big Data Technology CEUR Workshop Proceedings 1490 327337

[16] Burkov V N, Korgin N A and Novikov D A 2016 Problems of aggregation and decomposition mechanisms of management of organizational and technical systems Management issues 5 1423

[17] Lyubimov V V and Lashin V S 2017 External Stability of a Resonance during the Descent of a Spacecraft with a Small Variable Asymmetry in the Martian atmosphere Advances in Space Research 59(6) 1607-1613

[18] Lyubimov V V 2015 Numerical Simulation of the Resonance Effect at Re-entry of a Rigid Body with Low Inertial and Aerodynamic Asymmetries into the Atmosphere CEUR Workshop Proceedings 1490 198-210

[19] Dodonova N L and Kuznetsova O A 2017 About scarce resources allocation in conditions of incomplete information CEUR Workshop Proceedings 1904 130-134

[20] Geraskin M I and Kuznetsova O A 2017 Agents' Interaction algorithm in a strongly coupled system with a transferable utility CEUR Workshop Proceedings 2018 32-42

[21] Geraskin M I 2017 Algorithms of the information stimulation system of Russian citizens' sociooptimal actions CEUR Workshop Proceedings 1903 92-99

[22] Novikov D 2013 Theory of Control in Organizations (New York: Nova Science Publishers) p 341

[23] Kulikovskikh I M 2017 Anomaly detection in an ecological feature space to improve the accuracy of human activity identification in buildings Computer Optics 41(1) 126-133 DOI: 10.18287/2412-6179-2017-41-1-126-133 